\title{
Preparación para el trabajo: representaciones sociales del empleo y desempleo de jóvenes mexicanos sin trayectoria laboral
}

\section{Work preparation: Social representations of employment and unemployment of young Mexicans with no work experience}

\section{Preparação para o trabalho: Representações sociais do emprego e desemprego de jovens mexicanos sem padrões de trabalho}

\author{
Teresa Margarita Torres-López* \\ Martín Acosta-Fernández ${ }^{* *}$ \\ María de los Ángeles Aguilera-Velasco*** \\ Fecha de recepción: 29 de abril de 2016 \\ Fecha de evaluación: 18 de mayo de 2016 \\ Fecha de aceptación: 8 de mayo de 2017 \\ Disponible en línea: 18 de mayo de 2017
}

DOI: http://dx.doi.org/10.18359/reds.1867

Cómo citar este artículo:

Torres-López, T.M., Acosta-Fernández, M. y Aguilera-Velasco, M. A. (2017). Preparación para el trabajo: Representaciones sociales del empleo y desempleo de jóvenes mexicanos sin trayectoria laboral. Revista Educación y Desarrollo Social, 11(1), 85-101. DOI: http://dx.doi.org/10.18359/reds.1867.

Doctora en Antropología Social y Cultural, de la Universidad de Guadalajara. Centro Universitario de Ciencias de la Salud, Departamento de Salud Pública, Guadalajara, México. Correo electrónico: tere.torres.cucs@gmail.com

** Doctor en Ciencias de la Salud en el Trabajo, de la Universidad de Guadalajara. Centro Universitario de Ciencias de la Salud, Departamento de Salud Pública, Guadalajara, México. Correo electrónico: fmartin63@gmail.com

*** Doctora en Ciencias de la Salud en el Trabajo, de la Universidad de Guadalajara. Centro Universitario de Ciencias de la Salud, Departamento de Salud Pública, Guadalajara, México. Correo electrónico: aaguileracd@hotmail.com 


\title{
Resumen
}

Este trabajo forma parte de un proyecto de investigación en torno a las concepciones culturales de los conceptos de empleo y desempleo de diferentes grupos poblacionales de países iberoamericanos. Aquí se presenta el caso de los jóvenes estudiantes de bachillerato de Guadalajara, México, sin trayectoria laboral. El objetivo es identificar las representaciones sociales de empleo y desempleo, con el fin de generar elementos que permitan considerar su visión en el diseño de programas de apoyo para el empleo. El estudio fue cualitativo y se apoyó en métodos asociativos para la recolección del contenido de las representaciones sociales. La metodología del enfoque estructural de las representaciones sociales considera a representaciones como un conjunto de cognemas (términos del vocabulario que las personas utilizan para referirse a un objeto social determinado) organizados por múltiples relaciones que pueden ser orientadas y sistematizadas. Se concluye que las representaciones sociales del empleo y desempleo muestran una serie de expectativas que difícilmente llegarán a coincidir con la realidad a la que se enfrentarán los jóvenes en su proceso de inserción laboral.

Palabras clave: educación y empleo, empleo de jóvenes, desempleo, joven, transición a la vida activa.

\begin{abstract}
This work is part of a research project regarding cultural conceptions of the employment and unemployment concepts of different population groups from Latin American countries. Here we present the case of young high school students in Guadalajara, Mexico, with no work experience. The aim is to identify the social representations of employment and unemployment to generate elements that allow considering their vision for the design of support programs for employment. The study was qualitative and relied on associative methods for the collection of the content of social representations. The methodology of the structural approach of social representations considers representations as a set of cognemas (terms of vocabulary that people use to refer to a particular social object) organized by multiple relationships that can be oriented and systematized. It is concluded that the social representations of employment and unemployment show a set of expectations that will hardly match the reality that young people will face in their process of labor insertion.
\end{abstract}

Keywords: education and employment, youth employment, unemployment, youth, transition to working life.

\section{Resumo}

Este trabalho faz parte de um projeto de pesquisa sobre as concepções culturais de conceitos de emprego e desemprego de diferentes grupos populacionais de países ibero-americanos. Aqui é apresentado o caso dos jovens estudantes do ensino médio em Guadalajara, no México, sem trajetória de trabalho. O objetivo é identificar as representações sociais de emprego e desemprego, a fim de gerar elementos que permitam considerar a sua visão na concepção de programas de apoio para o emprego. O estudo foi qualitativo e foi baseado em métodos associativos para a colheita do conteúdo das representações sociais. A metodologia da abordagem estrutural das representações sociais considera a representações como um conjunto de cognemas (termos de vocabulário que as pessoas usam para se referir a um objeto social determinado) organizados por múltiplas relações que podem ser direcionadas e sistematizadas. Conclui-se que as representações sociais do emprego e desemprego mostram um conjunto de expectativas que dificilmente vêm para coincidir com a realidade que os jovens enfrentam no seu processo de inserção laboral.

Palavras-chave: educação e emprego, emprego de jovens, desemprego, jovem, transição para a vida ativa. 


\section{Introducción}

Desde el inicio de 2012 el mundo se encontraba ante un grave problema de desempleo y un déficit generalizado de trabajo decente. Después de tres años de una situación de crisis continua en los mercados de trabajo del mundo, y ante la perspectiva de un mayor deterioro de la actividad económica, hubo un retraso en el empleo mundial equivalente a 200 millones de puestos de trabajo; un incremento de 27 millones desde el inicio de la crisis (Organización Internacional del Trabajo [OIT], 2012a).

La crisis en cuestión de empleo se ha reflejado con especial dureza en los jóvenes. En 2011, 74,8 millones de jóvenes en edades comprendidas entre los 15 y los 24 años estaban desempleados, 4 millones más que en 2007. La tasa de desempleo juvenil mundial, del 12,7\%, sigue situada en un punto porcentual por encima del nivel anterior a la crisis. A escala mundial, los jóvenes tienen casi tres veces más probabilidades de estar desempleados que los adultos (OIT, 2012a).

La transición lenta e incierta de la escuela al trabajo genera más dificultades para la inserción en el mercado laboral, derivadas de la falta de experiencia. En este contexto han aumentado las pasantías, los aprendizajes profesionales y otras modalidades de adquisición de experiencia laboral con el fin de obtener trabajo decente. Sin embargo, dichos mecanismos pueden correr el riesgo, en algunos casos, de ser utilizados como una forma de obtener mano de obra barata o de reemplazar a otros trabajadores que ya están en la empresa (OIT, 2012b).

En América Latina y el Caribe viven 104,2 millones de personas de 15 a 24 años de edad, de los cuales cerca de 50 millones se encuentran trabajando o están buscando activamente empleo. Los jóvenes de ambos sexos enfrentan una situación difícil al incorporarse al mercado de trabajo, por falta de experiencia laboral y un contexto económico que no genera suficientes puestos de trabajo decente. La tasa de desempleo juvenil supera largamente a la adulta y los empleos que estos consiguen son más precarios y de menor calidad.

En la región, un total de 6,7 millones de jóvenes están desempleados, es decir, buscan empleo y no lo obtienen, una cifra que representa el $44 \%$ del total de desocupados en América Latina. Las mujeres jóvenes son las más perjudicadas: el porcentaje de las que están desempleadas (16,8\%) es casi 5 puntos porcentuales superior al de los hombres (12,2\%) (OIT, 2013, p. 86).

Las críticas condiciones socioeconómicas a las que estamos asistiendo en la actualidad están situando a la juventud en una posición social de marginación y precariedad crecientes, de resultados cada vez más preocupantes e insospechados. Ello a pesar de que el trabajo sigue ocupando una posición central para la mayor parte de componentes de las sociedades modernas. Asimismo, continúa ejerciendo de eje vertebrador 
de creencias, actitudes, identidades y otro tipo de opciones fundamentales (Agulló, 2014).

Es por ello que es necesario acercarse al punto de vista de la población en edad productiva para conocer cuál es su punto de vista en torno al tema del empleo y desempleo, en particular de los jóvenes. Los significados que estos le otorgan al trabajo, así como las atribuciones e imágenes permitirán acceder a su mundo de significados, además de sus propuestas ante la problemática que viven en torno a su inserción laboral.

Algunos estudios se han enfocado en el significado del trabajo, este consiste en un conjunto de creencias, valores y actitudes (de las personas) hacia el trabajo (que poseen las personas). Este significado se aprende antes (socialización para el trabajo) y durante el proceso de socialización en el trabajo y varía en función de las experiencias subjetivas y de aspectos situacionales que se producen en el contexto laboral y organizacional (Dakduk et al., 2008).

Otro enfoque ha sido el de destacar los valores laborales, definidos como aquellos aspectos o características del trabajo que son de importancia para una persona y que preferiría encontrar en su trabajo (De Jesús y Ordaz, 2006, p. 67). En el caso específico de los adolescentes, el trabajo está vinculado con vivencias emocionales positivas correlacionadas con la satisfacción de necesidades de valoración y reconocimiento, lo cual le otorga al individuo independencia material y psicológica (De Jesús y Ordaz, 2006).

Otro enfoque teórico que permite el acercamiento a los significados del empleo y desempleo son las representaciones sociales. Abric (2001) las define como un conjunto organizado y jerarquizado de juicios, actitudes e informaciones que un grupo social ha elaborado a propósito de un objeto social. Este objeto es reconstruido en un sistema simbólico que es interiorizado por los miembros de un grupo, que lo reproduce y comparte. Así, las representaciones sociales funcionan como un sistema de interpretación de la realidad, que rige las relaciones de los individuos con su entorno físico y social, además de que determina sus comportamientos o sus prácticas (Abric, 2001).

Una de las funciones sociales de las representaciones sociales es hacer convencionales los objetos, personas y eventos que se encuentran en la vida cotidiana; de tal forma que se establecen maneras de conocimiento distintivas y a la vez compartidas por un grupo cultural determinado. Por ello propician la comunicación, promueven el pensamiento colectivo y la identidad social. Además justifican las decisiones y conductas que se manifiestan en las interacciones sociales (Materán, 2008).

En un estudio sobre valores del trabajo y representaciones sociales realizado con estudiantes universitarios de Francia (de las carreras de educación a distancia, Psicología y Economía), se resalta a la 
inserción del individuo en su lugar de trabajo con el reconocimiento identitario y la autonomía implicados, así como la seguridad material, la inserción comunitaria en el mundo y las libertades sindicales en primer lugar, y a las garantías políticas en la organización como las de menor importancia. Se encontró, además, que la centralidad del concepto se ubica en el dinero y el salario, con algunos diferenciales de acuerdo con la carrera de estudios; los de educación a distancia resaltaron también la restricción y la expansión personales, mientras que los de Psicología, el placer de trabajar en lo que les gusta (Márquez et al., 2005).

Otro estudio realizado también con estudiantes universitarios, pero en Venezuela, destaca el el valor instrumental del trabajo como medio importante para el logro de múltiples fines, alcanzar ingresos y satisfacer necesidades. Como el valor expresivo, con la necesidad de ser independiente, autónomo, autosuficiente y asumir responsabilidades de adultos. Sin embargo, los participantes no mostraron claridad en cuanto al trabajo productivo decente, como es la conciencia de los derechos laborales fundamentales, la seguridad social y otros aspectos legales (Pereira, 2013).

No se encontraron estudios sobre las representaciones sociales de jóvenes sin trayectoria laboral; de ahí la importancia de conocer la mirada de estos como unos de los principales actores implicados en el proceso de transición de la escuela al trabajo.
Con base en estas ideas, el objetivo que guía este estudio es identificar las representaciones sociales de los conceptos de empleo y desempleo de estudiantes de bachillerato que aún no han realizado trabajo remunerado, a fin de generar elementos que permitan considerar su visión en el diseño de programas de apoyo para el empleo.

\section{Metodología}

\section{Diseño del estudio}

El estudio fue cualitativo y se apoyó en métodos asociativos para la recolección del contenido de las representaciones sociales. La metodología del enfoque estructural de las representaciones sociales considera a las representaciones como un conjunto de cognemas (términos del vocabulario que las personas utilizan para referirse a un objeto social determinado) organizados por múltiples relaciones que pueden ser orientadas y sistematizadas.

Las fases metodológicas que llevan a este conocimiento son:

1) La obtención de información para identificar el contenido de las representaciones sociales; los métodos utilizados son los interrogativos y los asociativos, como es el listado libre.

2) La descripción de la organización y la estructura de las representaciones sociales. Se utilizó el método de identificación de los lazos entre los elementos de las representaciones sociales llamado comparación pareada (Abric, 2001). 


\section{Participantes}

La población participante fueron estudiantes de bachillerato de una universidad pública de Guadalajara, México. La muestra fue no aleatoria de tipo propositivo (Suri, 2011). Los criterios para su selección fueron: estudiantes de bachillerato vigentes, mujeres y hombres, y habitantes de la ciudad de Guadalajara. Los participantes fueron 76 estudiantes de bachillerato habitantes de Guadalajara, México; sus edades fluctuaban entre 15 y 17 años, con un promedio de edad de 16,3 años; 36 fueron mujeres y 40 hombres, y ninguno de ellos había desarrollado trabajo remunerado.

\section{Obtención de información}

Las técnicas e instrumentos de recolección de datos fueron dos:

1) Listados libres: la técnica consiste en proporcionar a los sujetos una palabra o término inductor y pedirles que escriban las primeras palabras que se les ocurran con relación a la palabra inductora (en este caso las palabras empleo y desempleo). La principal ventaja de este procedimiento es que favorece la expresión más espontánea de los sujetos y por ello se espera que el contenido evocado esté más libre de racionalizaciones, sesgos de defensa o deseabilidad social (Bernard, 2006).

2) Cuestionario de comparación de pares: con las respuestas más frecuentes (obtenidas en los listados libres) se elabo- raron dos cuestionarios de comparación de pares, uno para el concepto de empleo y otro para desempleo. Este segundo instrumento permite a los entrevistados hacer un jueceo sobre los términos seleccionados, mediante la asociación de los dos términos que a su juicio consideren que son los más característicos de empleo (y desempleo) y que hagan una marca (con una letra A) en ellos. De los elementos restantes, elijen dos elementos que a su juicio consideran que son los menos característicos de empleo (y desempleo) y los marquen (con una letra B). Se continúa hasta que se hayan marcado los diez términos (con letras $C$, D y E). Así, al final se cuenta con cinco pares de asociaciones (Abric, 2001).

\section{Análisis de la información}

El análisis de datos fue diferente para cada una de los instrumentos utilizados:

1) Listados libres: luego de obtener las listas de palabras, se tabularon los términos por frecuencia de mención de empleo y desempleo. Se escogieron las diez palabras más frecuentes de cada lista para generar el instrumento de cuestionario de pares, aplicado en la segunda fase del estudio (uno para empleo y otro para desempleo). Un segundo análisis fue la categorización de las definiciones dadas para cada palabra, lo cual se hizo con análisis de contenido temático (Minayo, 2009).

2) Cuestionario de comparación de pares: los datos fueron analizados por 
medio de la obtención del índice distancia, que evalúa la relación de similitud y la relación de antagonismo o de exclusión. Además, mide la intensidad de la diferencia observada entre dos elementos en una población dada, de tal forma que +1 es la similitud y -1 la exclusión máxima. Para ello, se asignó un valor numérico a los pares de palabras seleccionadas. La escala de evaluación fue de $0 \mathrm{a}+2$ en relación con la importancia asignada a la asociación $(A=+2, B=-2, C=+1, D=-1$ y $E=0)$ (Abric, 2001). Con estas puntaciones se calculó el análisis de distancia ${ }^{1}$ con el apoyo de una hoja de cálculo de Excel. Con los índices obtenidos en la relación de cada uno de los pares seleccionados se construyó un grafo. Esto es, una representación gráfica de la conexión de todos los cognemas. El procedimiento para su construcción proviene de la teoría de grafos y es descrita por Doise et al., (2005). El análisis de las asociaciones identificadas permite analizar las relaciones significativas de la naturaleza de las representaciones sociales del sujeto o de ciertas dimensiones de estas (Abric, 2001).

\section{Aspectos éticos}

A cada participante se le comunicó que las descripciones o referencias que se hicieran en el reporte de investigación

La fórmula utilizada para su obtención es: $\mathrm{D}=[2$ (en - sumatoria dj) $]-1, \mathrm{D}=$ distancia en observada, $\mathrm{n}=$ número de sujetos, e = escala. nunca evidenciarían datos personales que pusieran en riesgo su anonimato e integridad. Salvo su edad, escolaridad y género no se mencionaría otra información mediante la cual pudieran ser localizados o identificados, ya que no es de interés ni relevancia para esta investigación evidenciar a ninguna persona ni divulgar su identidad y localización. No hubo consentimiento escrito, ya que este puede ser expreso y se debe considerar el contexto de desarrollo de grupos culturales diversos al del investigador, sus tradiciones en cuanto a comunicación y decisión, y se deben respetar estos procedimientos (Aguilera-Guzmán et al., 2008) (las personas no acceden a estampar firmas con facilidad, por experiencias propias o ajenas, de fraudes y despojos). Además, el proyecto de investigación que fundamentó el presente estudio fue evaluado y en su momento aprobado por el comité de ética e investigación del Instituto de Investigación en Salud Ocupacional del Departamento de Salud Pública, de la Universidad de Guadalajara (registro IISO/CI/01-27/2013).

\section{Resultados}

\section{Contendido de las representacio- nes sociales del empleo}

Las categorías temáticas encontradas en las representaciones sociales de empleo se presentan en la tabla 1. 
Tabla 1. Categorías encontradas en el análisis de las palabras obtenidas en los listados libres para las representaciones sociales de empleo

\begin{tabular}{|l|c|c|}
\hline \multicolumn{1}{|c|}{ Categoría } & Frecuencia & Porcentaje \\
\hline $\begin{array}{l}\text { Elementos } \\
\text { necesarios }\end{array}$ & 23 & 26,0 \\
\hline $\begin{array}{l}\text { Beneficios } \\
\text { personales }\end{array}$ & 23 & 26,0 \\
\hline $\begin{array}{l}\text { Componente } \\
\text { económico }\end{array}$ & 19 & 21,6 \\
\hline $\begin{array}{l}\text { Aspectos } \\
\text { negativos }\end{array}$ & 9 & 10,4 \\
\hline $\begin{array}{l}\text { Beneficios } \\
\text { materiales }\end{array}$ & 8 & 9,2 \\
\hline $\begin{array}{l}\text { Beneficios } \\
\text { sociales }\end{array}$ & 88 & 100 \\
\hline \multicolumn{1}{|c|}{ Total } & 6,8 \\
\hline
\end{tabular}

Fuente: elaboración propia.

\section{Categorías}

1) Elementos necesarios: comprende los conocimientos y la preparación académica, así como los valores de responsabilidad, compromiso, dedicación, honestidad y madurez. Además de la actitud, esfuerzo, obligación y puntualidad requeridos para desempeñar un empleo.

2) Beneficios personales derivados de contar con un empleo: incluye los sentimientos de satisfacción, felicidad, alegría, tranquilidad y plenitud. Mas la certeza de sentirse productivo, con éxito, experiencia, superación, útil, independiente y valorado. Además, con las ventajas adicionales de realizar viajes y tener una vida saludable.

3) Componente económico: comprende todas las palabras relacionadas con el dinero, los ingresos y la buena economía.

4) Aspectos negativos de tener un empleo: tener todo el tiempo ocupado, aunado a la rutina que llevan al cansancio, estrés y la flojera.

5) Beneficios materiales producto de la percepción económica: por un lado, el poder, la prosperidad, la administración, los lujos y los ahorros; por el otro, los gastos y las necesidades.

6) Beneficios sociales: son los de realizar trabajo en equipo, tener amigos y un futuro familiar, además de desarrollar la tolerancia hacia otras personas.

\section{Organización y estructura de las re- presentaciones sociales de empleo}

En la figura 1 se presenta el grafo de las representaciones sociales de empleo de los participantes. Las palabras que obtienen mayores puntuaciones son felicidad (un chico comentó: "puedo hacer lo que me gusta") y responsabilidad (una chica dijo: "un empleo requiere organización y honestidad para desempeñarlo correctamente"). Se pueden observar dos agrupaciones de palabras (el corte se establece en las puntuaciones más bajas obtenidas en los índices de distancia): la primera comprende satisfacción ("me siento bien conmigo mismo"), unido a 
Preparación para el trabajo: representaciones sociales del empleo y desempleo de jóvenes mexicanos...

felicidad y esta a su vez, a éxito ("al hacer bien tu trabajo puedes ser una persona exitosa en lo que te desempeñes"). El cognema felicidad (la cual funciona de unión con el siguiente grupo de palabras) está unido a madurez ("cuando tienes un empleo es señal de que comienzas a sustentar algunos de tus gastos"), de la cual se desprenden conocimiento ("sin conocimiento no puedes trabajar") y superación ("voy creciendo por mí mismo"). El segundo grupo incluye de forma secuencial experiencia ("te da confianza y ganas conocimiento"), dinero ("te da un sostén económico y es lo principal de un trabajo"), responsabilidad y tiempo ("no tienes suficiente tiempo libre").

\section{Contendido de las representaciones sociales del desempleo}

Las categorías temáticas encontradas en las representaciones sociales de desempleo se presentan en el tabla 2.

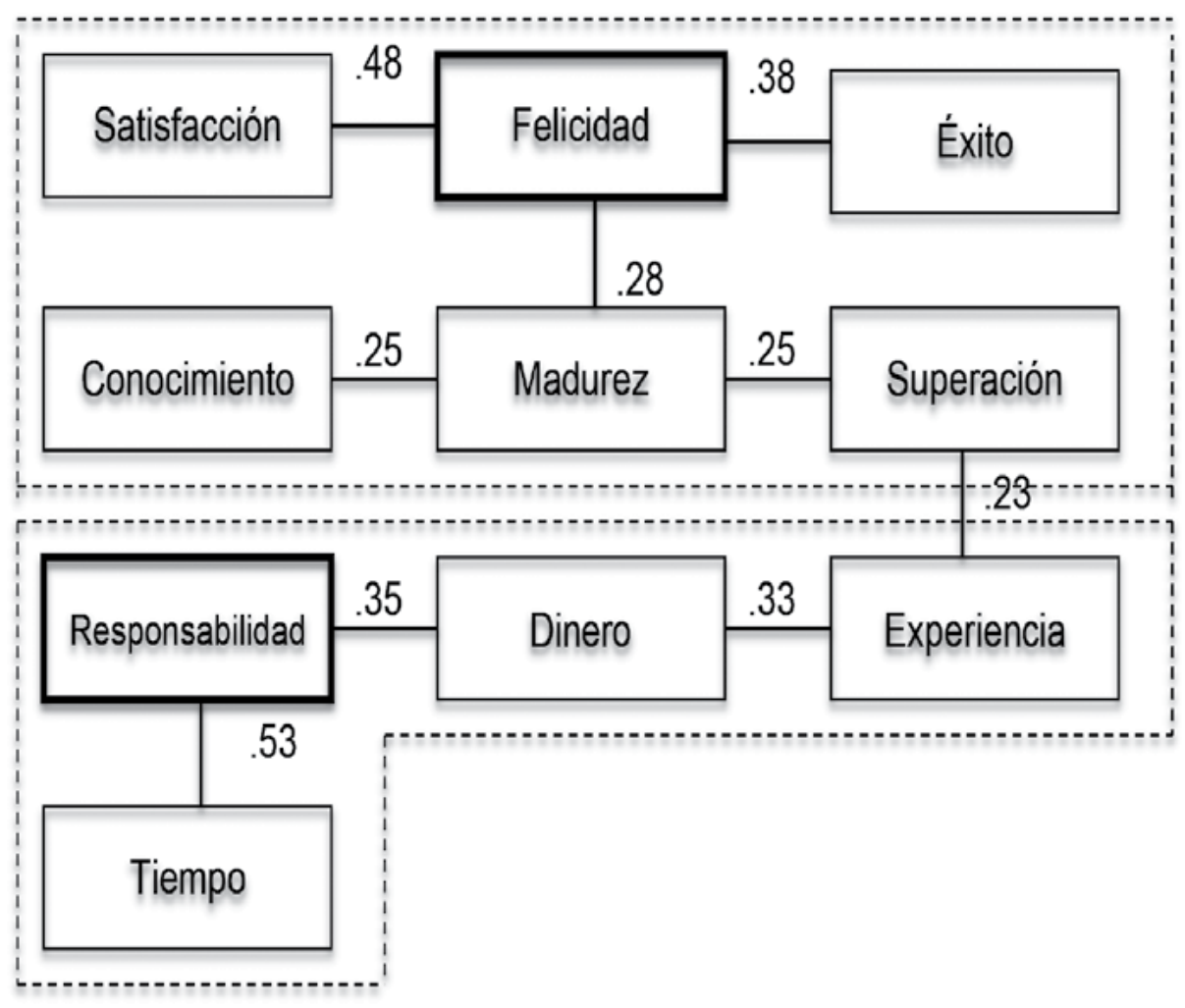

Figura 1. Representaciones sociales de empleo de los participantes

Fuente: elaboración propia.

Nota: los decimales entre las palabras corresponden al índice de distancia encontrado en cada asociación. 
Tabla 2. Categorías encontradas en el análisis de la palabras obtenidas en los listado libres para las representaciones sociales de desempleo

\begin{tabular}{|l|c|c|}
\hline \multicolumn{1}{|c|}{ Categoría } & Frecuencia & Porcentaje \\
\hline $\begin{array}{l}\text { Componente } \\
\text { económico }\end{array}$ & 20 & 22,7 \\
\hline $\begin{array}{l}\text { Efectos } \\
\text { personales }\end{array}$ & 20 & 22,7 \\
\hline Efectos sociales & 21 & 23,8 \\
\hline $\begin{array}{l}\text { Emociones } \\
\text { negativas }\end{array}$ & 16 & 18,3 \\
\hline $\begin{array}{l}\text { Elementos } \\
\text { asociados }\end{array}$ & 11 & 12,5 \\
\hline Total & 88 & 100 \\
\hline
\end{tabular}

Fuente: elaboración propia.

\section{Categorías}

1) Componente económico: comprende lo relacionado con la falta de dinero, pérdida y pobreza.

2) Efectos personales derivados del desempleo: incluye los sentimientos de fracaso, crisis, impotencia, inutilidad, mediocridad, dependencia y conformidad, lo que lleva a la enfermedad, el dolor, la falta de amigos y de expectativas, considerar el suicidio y a no desarrollar habilidades, experiencia ni conocimientos.

3) Efectos sociales derivados del desempleo: son la existencia de necesidades, hambre, carencias, ignoran94 cia, racismo y las consecuencias de vagancia, inseguridad, narcotráfico y robo.
4) Emociones negativas producto del desempleo: incluye tristeza, depresión, desánimo, enojo, rabia, desesperación, estrés y frustración.

5) Elementos asociados: como son el ocio y el desperdicio de tiempo, dificultades de acceso al trabajo y a México como un ejemplo de desempleo.

\section{Organización y estructura de las representaciones sociales de desempleo}

En la figura 2 se presentan las representaciones sociales de desempleo de los participantes. Las dos palabas con mayor puntuación son necesidad (una chica comentó: "necesidad de mantener una familia o de pagar deudas") y ocio (un joven dijo: "no haces nada y no tienes ninguna responsabilidad"). Se pueden observar dos agrupaciones de palabras: la primera es pobreza ("no puedes comprar las cosas que deseas, es más difícil cumplir tus metas"), frustración ("por no cumplir tus sueños y metas, empiezas a frustrarte por no saber cuándo lo lograrás"), necesidad y estrés ("no sabes qué te depara el futuro"). El cognema necesidad funciona como unión con el siguiente grupo de palabras conformado por hambre ("las familias a veces no tienen para comprar comida"), tristeza ("Porque eres dependiente de alguien más y muchas veces el desempleo no es algo que depende de uno"), enojo ("me enojo por la falta de dinero") y ocio. 
Preparación para el trabajo: representaciones sociales del empleo y desempleo de jóvenes mexicanos...

De esta última palabra se desprenden dependencia ("dependes de otras personas para todo lo que necesitas") y delincuencia ("no hay trabajo, no dinero, no comida, gente pobre, niños con hambre, asaltan, roban, etc.").

\section{Discusión y conclusiones}

\section{El empleo:}

\section{felicidad y responsabilidad}

Las representaciones sociales de empleo son las de una ocupación que requiere contar con los elementos necesarios tanto de preparación académica, como de valores (responsabilidad, compromiso, honestidad, etc.) y esfuerzo personales (obligación, puntualidad, entre otros), lo que derivará en la obtención de un ingreso económico y de bienes materiales, además de beneficios más en lo personal (emociones positivas, ser productivo, exitoso e independiente, etc.) y menos en lo social (trabajo en equipo, amigos, tolerancia y demás).

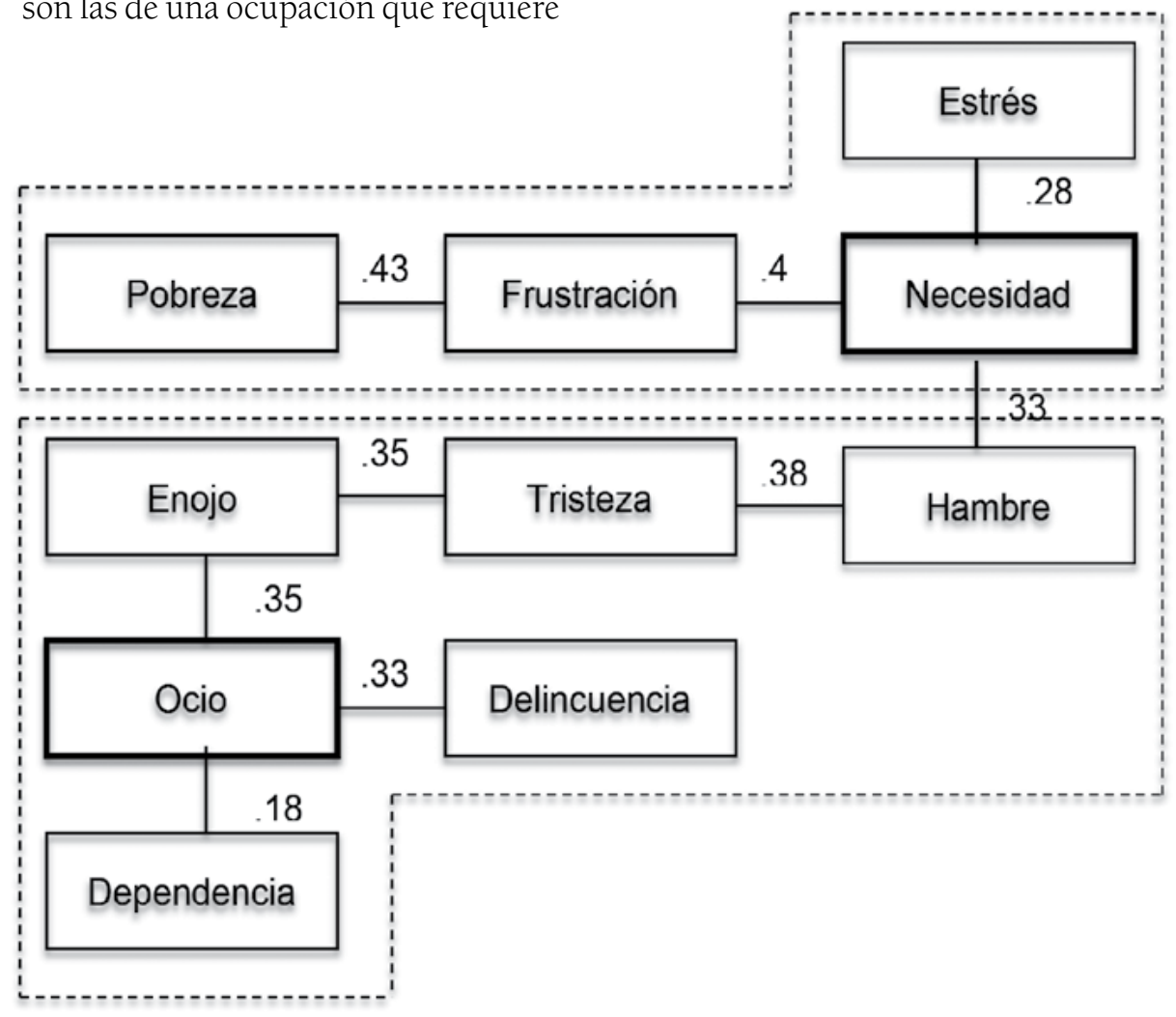

Figura 2. Representaciones sociales de desempleo de los participantes

Fuente: elaboración propia.

Nota: los decimales entre las palabras corresponden al índice de distancia encontrado en cada asociación. 
Implica también algunos aspectos negativos, como el tiempo ocupado, la rutina y el cansancio. El énfasis principal se encuentra en la felicidad y la responsabilidad.

Los participantes identificaron el empleo como una estrategia o medio de aprendizaje en la que se valida y refuerza a la persona, y se concibe como una forma para demostrar la utilidad del joven mismo, haciendo con su labor una contribución pertinente a su entorno (De Jesús y Ordaz, 2006). Además, su asociación con la independencia que se obtiene al desarrollarlo es vista como "[...] un lugar de expansión en el que se rescata su carácter de construcción, comparándose con un hogar, una actividad demandante de potencialidades y habilidades en la que no sólo te validas, sino que también creas tu espacio" (De Jesús y Ordaz, 2006, p. 69). Por tanto, el empleo es concebido como una actividad que permite la satisfacción de necesidades sociales y psicológicas, además que potencia valores personales como la competencia y el dominio en áreas académicas y de habilidades específicas (Valls y Martínez, 2004).

Esta concepción del trabajo coincide con el sistema de creencias humanistas encontrado por Sidani y Jamali (2010) en torno al trabajo desde la visión de trabajadores egipcios. Es asociado a una perspectiva de crecimiento individual 96 y de desarrollo a través del empleo, lo que refleja una apreciación importante y gran significado del trabajo.
Llama la atención la importancia que tiene para los jóvenes la preparación académica; esto ha sido resaltado como un aspecto central para el acceso al empleo y para el desempeño laboral. El aumento en la cobertura escolar y la extensión de los años de escolaridad de la población en la región, tanto en el nivel básico o primario como en la escuela media, es positivo. Si bien esto ya no garantizan empleos formales o "de calidad", sí les permite tener prioridad con respecto a quienes poseen menos años de escolaridad (Isacovich, 2015).

De acuerdo con un estudio realizado por Reyes-Terrón y Elizarrás-Hernández, (2013) en relación con jóvenes del Estado de México (México) que no estudian ni trabajan, se encontró que a mayor escolaridad menor es la existencia de estos en las estadísticas nacionales. El grado de escolaridad está determinando la posición en el trabajo y en las ocupaciones de las personas, ya que el número de individuos con estudios de maestría y doctorado no representa ni siquiera el 0,04\% del total de sujetos que ni estudian ni trabajan. Esto lleva a poner mayor atención en aquellas personas que deciden no continuar con estudios posteriores a los niveles de primaria, secundaria, media superior o carrera técnica, dado que aparecen como los grupos con mayor vulnerabilidad al fenómeno estudiado, pues presentan un mayor crecimiento (Reyes-Terrón y Elizarrás-Hernández, 2013). 
Aún cuando los participantes incluyeron elementos negativos del empleo, estos no contienen referencias de su desarrollo en condiciones de precariedad. Al igual que los estudiantes universitarios de Venezuela (Pereira, 2013) no consideran sus derechos laborales ni seguridad social; en suma, lo que se ha denominado trabajo decente desde la perspectiva de la OIT (2012c), con salarios dignos, futuro estable, protección social y acceso a derechos fundamentales.

Finalmente, la centralidad de las palabras felicidad y responsabilidad destaca la importancia que tiene el trabajo, ya que no es solo una fuente de ingresos económicos, sino que también se relaciona con el bienestar psíquico y social (Valls y Martínez, 2004).

\section{El desempleo entre el ocio y la necesidad económica}

Las representaciones sociales de desempleo son de un tiempo desocupado que implica la falta de dinero y la vivencia de pobreza, con efectos personales (sentimientos negativos de fracaso, crisis, impotencia, dependencia y demás), sociales (carencias, hambre, inseguridad, etc.) que genera emociones negativas (tristeza, desánimo, enojo, frustración, entre otras). El énfasis del concepto esta en las palabras necesidad y ocio.

Los efectos que tendría para los jóvenes el no contar con un empleo los ubican en un contexto social que no les permite tomar decisiones relevantes, debido a su condición de dependencia. "Lo anterior ocasiona que no exista la posibilidad, ni las opciones, de construir un proyecto de vida particular, propio, autónomo, ya que no se ha cumplido plenamente el proceso de transición de la persona joven al mercado de trabajo" (García-García, 2015, p. 1226).

Como lo indica Pedraza (2008), la falta de trabajo decente a temprana edad afecta las perspectivas laborales futuras y disminuye la capacidad de ahorro a lo largo del ciclo vital. El vínculo entre el desempleo juvenil y la exclusión social podría generar sentimientos de marginalización y aumentar los grados de violencia y las adicciones. Además, la juventud desempleada o subempleada no contribuye a la economía y hay un desperdicio de capital humano que está en pleno desarrollo físico y mental.

Por lo tanto, la transición a la vida activa, de la escuela al trabajo, no se presenta de forma lineal. "La juventud, como estadio psicosociológico transitorio, ha dejado de ser un paréntesis que se completa con la inserción socio laboral" (Moral-Jiménez, 2007, p. 175). La incertidumbre sobre el futuro generaría un problema con consecuencias psicosociales en los procesos de construcción de la identidad (Moral-Jiménez, 2007). Por ello, las transiciones parecen estar caracterizadas por situaciones ambivalentes, ya no se desarrollan linealmente desde una dependencia social hasta una independencia total (Otero, 2010). 
Estas representaciones sociales del desempleo tienen su fundamento en la crisis estructural que atraviesa México y las sociedades capitalistas en general, lo que está afectando e incidiendo de forma significativa en el proceso de inserción social de los jóvenes. La precariedad del empleo afecta principalmente a los más jóvenes (OIT, 2013). Dicha crisis socioeconómica está provocando una prolongación y un retraso en las prácticas de transición a la sociedad adulta. Como lo indicó De Oliveira (2006), tomando como fuente la Encuesta Nacional de la Juventud 2000 de México, a pesar de la heterogeneidad sociodemográfica, la mayoría de los jóvenes asalariados se inserta en los mercados de trabajo en empleos que se caracterizan por grados de precariedad que van de moderados a muy altos (solo un tercio de ellos desempeña actividades no precarias o de baja precariedad).

Lo que pone de manifiesto la situación de vulnerabilidad laboral y social a la que están sujetos los jóvenes, y enfrentan situaciones de gran incertidumbre en el mundo laboral, ya sea por la falta de protección y estabilidad laboral o por los bajos salarios que perciben (De Oliveira, 2006).

Finalmente, la centralidad de las palabras ocio y necesidad destaca la incertidumbre de un tiempo desocupado que no permite satisfacer las necesidades 98 fundamentales, en concordancia con el valor instrumental del trabajo citado por Pereira (2013) y la seguridad material encontrada por Márquez et al. (2005).
Las representaciones sociales del empleo y desempleo muestran una serie de expectativas que difícilmente llegarán a coincidir con la realidad a la que se enfrentarán los jóvenes en su proceso de inserción laboral, máxime cuando las opciones que se presentan en el contexto mexicano en torno a la preparación para el empleo son limitadas. En el caso de las iniciativas dirigidas a sujetos jóvenes pobres de Argentina, Isacovich (2015) destaca que algunas de ellas contribuyen a anclarlos en sus contextos de exclusión, al proponerles una educación de baja calidad orientada a empleos informales e inestables. Mientras que Pieck (2001) señala la poca presencia de instituciones no gubernamentales en el campo de la formación de jóvenes, ya que la oferta total corresponde a las instituciones del Gobierno en el caso del estado de México, situación que parece ser general en todo el país. Esto muestra el poco interés de la sociedad civil por este ámbito de la educación y evidencia la ausencia de fórmulas alternativas que busquen desde otras ópticas incidir en este nivel. Indica, además, que existen algunas organizaciones no gubernamentales (ONG) que trabajan con jóvenes, cuyo interés se ubica más bien en el ámbito social y cultural, y no hay interesadas en el tema de la capacitación laboral de la juventud (Pieck, 2001).

En suma, las representaciones sociales de empleo y desempleo parecen mostrar una relación complementaria, ya que se identificaron las mismas categorías de análisis para ambos conceptos. Una de 
las funciones de las representaciones sociales es la conformación de identidades personales y sociales, lo que permite la expresión y la configuración de grupos (Materán, 2008). El peligro que se corre es que la crisis del empleo juvenil podría generar en los jóvenes identidades laborales inestables y vulnerables con poca involucración y compromiso personal y social.

Una de las aportaciones metodológicas de este estudio es que propone una forma de acercamiento científico a las representaciones sociales de la población con estrategias de recolección de información sencilla y más rápida que las entrevistas tradicionales. Esto permite el examen de gran cantidad de datos de forma coherente y accesible, facilita la comparación entre subgrupos de forma flexible y provee información sobre representaciones culturales que pueden condicionar prácticas. Otra es el haber destacado el punto de vista de una población que pocas veces es tomada en cuenta para el establecimiento de programas de apoyo para el empleo, esta es, la población adolescente y joven que ingresa de forma masiva al desarrollo de actividades remuneradas.

\section{Referencias}

Abric, J.C. (2001). Prácticas y representaciones sociales. México, D. F.: Ediciones Coyoacán.

Aguilera-Guzmán, R.S., Mondragón, L. y Medina-Mora, M.E. (2008). Consideraciones éticas en intervencio- nes comunitarias: la pertinencia del consentimiento informado. Salud Mental, 31, pp. 129-138.

Agulló, E. (2014). Jóvenes, trabajo e identidad. Oviedo: Universidad de Oviedo.

Bernard, R. (2006). Research Methods in Anthropology. Oxford: Altamira Press.

De Jesús, M. y Ordaz, M.G. (2006). El significado del trabajo: estudio comparativo entre jóvenes empleados y desempleados. Segunda Época, XXV(2), pp. 64-77.

Dakduk, S., González, A. y Montila, V. (2008). Relación de variables spocopdemográficas, psicológicas y la condición laboral con el significado del trabajo. Revista Interamericana de Psicología, 42(2), 390-401.

De Oliveira, O. (2006). Jóvenes y precariedad laboral en México. Papeles de Población, 49 , pp. 37-73.

Doise, W., Clemence, A. y Lorenzi-Cioldi, F. (2005). The quantitative analysis of social representations. London: Harvester Wheatsheaf.

García-García, V.D. (2015). Emprendimiento empresarial juvenil: una evaluación con jóvenesestudiantes de universidad. Revista Latinoamericana de Ciencias Sociales, Niñezy Juventud, 13(2), 1221-1236. DOI: 10.11600/1692715x.13246200315

Isacovich, P. (2015). Políticas para la inserción laboral de jóvenes: estudios en Latinoamérica y Argentina. Revista Latinoamericana de Ciencias Sociales, Niñezy Juventud, 13(2), 893-905. DOI: 10.11600/1692715x.13224120214.

Márquez, E., Friemel, É. y Rouquette, M.L. (2005). Valores del trabajo y 
representaciones sociales. Un estudio exploratorio. Trayectorias, VII(18), 17 32.

Materán, A. (2008). Las representaciones sociales: un referente teórico para la investigación educativa. Geoenseñanza, 13(2), 243-248.

Minayo, C. (2009). La artesanía de la investigación cualitativa. Buenos Aires: Lugar Editorial.

Moral-Jiménez, M.V. (2007). Preparación para el trabajo de los jóvenes contemporáneos en una sociedad postindustrial: trabajo, educación y globalización. Estudios sobre Educación, 13, 171-194.

Organización Internacional del Trabajo [OIT] (2012a). Tendencias mundiales del empleo 2012. Prevenir una crisis mayor del empleo. Recuperado el 28 de octubre de 2015, de http://www.ilo.org/wcmsp5/groups/ public/--dgreports/-dcomm/-publ/documents/publication/ wcms_168095.pdf

Organización Internacional del Trabajo [OIT]. (2012b). La crisis del empleo juvenil: un llamado a la acción. Resolución y conclusiones de la 101 ${ }^{a}$ reunión de la Conferencia Internacional del Trabajo. Recuperado el 28 de octubre de 2015, de http://www.ilo.org/ wcmsp5/groups/public/—ed_norm/— relconf/documents/meetingdocument/ wcms_187080.pdf

Organización Internacional del Trabajo [OIT] (2012c). Del trabajo precario al trabajo decente: documento final del simposio de los trabajadores sobre politicas y reglamentación para luchar contra el empleo precario. Recuperado el 28 de octubre de 2015, de http://www.ilo. org/wcmsp5/groups/public/_-ed.../ wcms_179789.pdf

Organización Internacional del Trabajo [OIT] (2013). Trabajo decente e igualdad de género. Políticas para mejorar el acceso y la calidad del empleo de las mujeres en América Latina y el Caribe. Recuperado el 28 de octubre de 2015, de http:// www.ilo.org/wcmsp5/groups/public/americas/--ro-lima/_-sro santiago/ documents/publication/wcms_233161. pdf

Otero, A.E. (2010). Los avatares de la transición a la vida adulta, el papel de la educación y el trabajo en los recorridos juveniles. Margen, 59, 1-13.

Pedraza, A.C. (2008). El mercado laboral de los jóvenes y las jóvenes de Colombia: realidades y respuestas políticas actuales. Revista Latinoamericana de Ciencias Sociales, Niñezy Juventud, 6(2), 853-884.

Pereira, L. (2013). La representación social del trabajo en jóvenes universitarios. Revista Latinoamericana de derecho Social, 17, 145-177.

Pieck, E. (2001). La capacitación para jóvenes en situación de pobreza. El caso de México. En E. Pieck. (Ed.), Los jóvenes y el trabajo: la educación frente a la exclusión social (pp. 95-154). México: Universidad Iberoamericana.

Reyes-Terrón, A. M. y ElizarrarásHernández, M. (2013). Los jóvenes y las jóvenes en el Estado de México: sociodemografía y empleo 2010. Revista Latinoamericana de Ciencias Sociales, Niñez y Juventud, 11(1), 287-304. DOI: 10.11600/1692715x.11119060512. 
Preparación para el trabajo: representaciones sociales del empleo y desempleo de jóvenes mexicanos...

Sidani, Y.M. y Jamali, D. (2010). The Egyptian Worker: Work Beliefs and Attitudes. Journal of Business Ethics, 92, 433-450. DOI: 10.1007/s10551009-0166-1.

Suri, H. (2011). Purposeful Sampling in Qualitative Research Synthesis. Qualitative Research Journal, 11(2), pp.
63-75. DOI: http://dx.doi.org/10.3316/ QRJ1102063

Valls, F. y Martínez, J.M. (2004). La centralidad y el valor del trabajo en el proceso de inserción laboral de personas desempleadas. Revista de Psicología del Trabajo y de las Organizaciones, 20(3), 337-354. 